\title{
Estrategias de negociación e intervención entre agentes gubernamentales y actores locales en el marco del desarrollo alternativo $^{+}$
}

\author{
GABRIELA STÖCKLI* \\ Pontificia Universidad Católica del Perú \\ gabriela.stockli@pucp.pe \\ https://doi.org/10.18800/rcpg.201701.003
}

\begin{abstract}
RESUMEN
El artículo analiza las interacciones entre actores del Estado y la población objetivo en la implementación de un programa de desarrollo alternativo en el valle del Aguaytía (UcayaliPerú). La investigación postula que la implementación local de una política central se adecua a través de procesos informales de negociación que instauran ajustes y flexibilizaciones en la ejecución de las actividades del programa. Los acuerdos establecidos cumplen una función clave en la implementación, pues ayudan en la construcción de confianza entre los actores estatales y las comunidades locales. Finalmente, se discuten las limitaciones de la mirada tecnócrata en el diseño del programa y cómo, en un contexto de negociaciones informales, el énfasis excesivo en indicadores de desempeño puede llevar a la generación de indicadores poco confiables.

Palabras clave: políticas públicas, estrategias de negociación, capacidades estatales, desarrollo alternativo, legitimidad.
\end{abstract}

Negotiation and intervention strategies between government agents and local actors in the framework of alternative development

\section{Abstract}

This article analyzes the interactions between government actors and the target population of an Alternative Development program in the Aguaytía Valley, located in the Amazon Rainforest (Ucayali - Perú). The main objective is to understand how the implementation of a general policy in a local context leads to a series of adjustments and flexibilized practices through a process of informal negotiation between the actors. The findings prove that these informal agreements have a key role in making the program work at a local level by building a sense of trust between government actors and the local communities and making it more responsive to the local needs. Finally, we discuss the challenges and limitations of the technocratic approach in the design and implementation of this Alternative Development program, and how an

\footnotetext{
* Licenciada en Sociología por la Pontificia Universidad Católica del Perú. Es investigadora del Laboratorio de Criminología y Estudios sobre la Violencia de la PUCP.

+Recibido el 15 de febrero de 2017; aceptado el 13 de mayo de 2017.
} 
excessive focus on performance indicators, in a context of informal negotiations, leads to the institutionalization of vicious cycles and vacuous indicators.

Key words: public policies, negotiation strategies, state capacities, alternative development, legitimacy. 


\section{INTRODUCCIÓN}

El Perú es actualmente el segundo productor en el mundo de hoja de coca y de su derivado ilícito, la cocaína. Se estima que en 2015 la produción fue de 96304 toneladas métricas concentradas en un 69,5\% en el valle del ApurimacEne (UNODC, 2016) ${ }^{1}$. A pesar de los continuos esfuerzos que el Estado peruano viene relizando, la lucha contra el narcotráfico en el Perú aún tiene mucho por delante.

Como una de las principales medidas para luchar contra el narcotráfico, el Estado peruano viene concentrado sus esfuerzos en la erradicación de la hoja de coca a través del Ministerio del Interior. Sin embargo, según el planteamiento actual se considera que la erradicación en sí misma no es suficiente para disminuir sostenidamente la producción de hoja de coca, ya que también es necesario derivar la actividad agrícola hacia fines lícitos que generen desarrollo en los contextos afectados por la erradicación. Es aquí donde los programas de desarrollo alternativo (DA), que buscan fomentar el desarrollo económico y agrícola en las zonas cocaleras, generalmente en situación de pobreza, se hacen necesarios.

En tanto el éxito de lo planteado por el Estado se basa en la participación sostenida de las familias de agricultores excocaleros en el programa de DA, surge una dinámica delicada y sumamente compleja donde los actores estatales deben conseguir que los objetivos institucionales trazados a nivel central sean asumidos por la población intervenida (y los demás actores locales). Se convierte, así, en un reto de puentes comunicativos entre lo que se plantea como voluntad política y lo que es asumido como beneficioso para la población.

¿Cómo se establece la dinámica entre la intervención estatal (como lineamiento de política y como grupo de individuos concretos que la implementan) y la población objetivo que la recibe, tomando en consideración que todos los actores tienen intereses particulares? Este artículo presenta los principales hallazgos de una investigación de más de dos años de trabajo de campo y entrevistas a todos los actores involucrados ${ }^{2}$.

En la primera parte del documento contextualizamos el programa de DA dentro de la Estrategia Nacional de Lucha Contra las Drogas y el rol del valle de Aguaytía como lugar estratégico para implementar el proyecto modelo.

\footnotetext{
En el año 2013 el Perú era el principal productor de hoja de coca y de cocaína, pero la extensión de cultivos disminuyeron en 2015 un 6,1\% con respecto a 2014 (de 42900 hectáreas a 40 300).

2 Los resultados presentados acá son parte de la tesis para obtener el título de Licenciada en Sociología sostenida en el año 2013 (PUCP). Asimismo, en un periodo posterior se trabajó en un proyecto de desarrollo aternativo liderado por la cooperación internacional en otra región del Perú.
} 
En el segundo apartado expondremos los elementos teóricos que nos ayudan a abordar este caso desde la perspectiva de las capacidades estatales (Evans, 1996; Alonso, 2007; Oszlak y Orellana, 2001), enfatizando en la capacidad relacional del Estado (Repetto, 2003) como elemento central de la implementación de políticas públicas, que conforman la agenda de acuerdo a la visión que tiene el Estado. En el tercer apartado se describe brevemente la triangulación metodológica seguida para realizar la investigación del estudio de caso de Aguaytía. En la cuarta parte ahondamos en la idea que la capacidad relacional también focaliza la atención en un elemento central que permita la implementación de políticas a nivel local: la legitimidad del Estado a través de actores concretos en las zonas intervenidas (Andrews, Pritchett y Woolcock, 2016). Así, en esta parte, correspondiente al apartado analítico, analizamos la problemática de la intervención en base a la actuación de la voluntad política de los programas de desarrollo alternativo a nivel local. Se analizan las estrategias de negociación que utilizan los agentes estatales para intervenir y lograr estabilidad en las comunidades, encontrándose las siguientes: la individualización de la participación para impedir una respuesta colectiva, la flexibilización de la normativa con respecto a la siembra de cultivos de hoja de coca como forma de generar confianza y paliar efectos colaterales de la erradicación y, por último, el desplazamiento de responsabilidades ante los efectos negativos de la erradicación y de los anteriores programas de DA a otras instituciones (estatales y no estatales). Por último, cerramos el artículo con conclusiones y recomendaciones respecto a la forma de generar accountability tomando en cuenta que todos los actores tienen intereses particulares.

\section{Contextualizando la política del da y El ROL de AguaYtía}

Como fruto de la experiencia de desarrollo alternativo años anteriores con la cooperación internacional, el Estado peruano logró plantear los componentes y conceptualización del DA en la Estrategia Nacional de Lucha Contra las Drogas (2012-2016) en zonas post erradicadas (de hoja de coca). Se trata de un lineamiento central y orientador de la política de intervención de la Comisión Nacional para el Desarrollo y Vida sin Drogas (Devida) y, por lo tanto, es parte del marco institucional/normativo que organiza las acciones o roles de los actores en contextos de poserradicación. Según Devida, el DA:

[...] promueve el cambio de actitudes de la población hacia un desarrollo y vida lícita sin la influencia de la economía y las actividades delictivas del narcotráfico. Para tal fin fomenta el esfuerzo conjunto de la población, sus organizaciones y autoridades, con 
el apoyo del gobierno nacional, gobierno regional y gobiernos locales, con énfasis en el desarrollo del capital social de los ámbitos de intervención, asimismo incorporando el aporte de la cooperación internacional y la inversión privada, con el objetivo de lograr un desarrollo integral con inclusión social, que prioriza la gestión ambiental y el aprovechamiento racional de los recursos naturales (Devida, 2012, p. 43).

Las ideas fundamentales detrás del DA como medida de desarrollo para la lucha contra el narcotráfico serían las siguientes:

- El cambio de actitudes hacia un desarrollo de vida lícita

- El apoyo de los gobiernos subnacionales y sus organizaciones de base

- Desarrollo de capital social en las comunidades intervenidas

- Integración del aporte de la cooperación internacional

Las ideas anteriormente señaladas denotan un énfasis político en lograr territorios comprometidos con el enfoque de lucha contra las drogas que plantea el Estado, de modo que genere legitimidad y sostenibilidad a largo plazo (evitando así caer en el error de actuación limitada de un gobierno específico a través de una política puntual). La implementación en los espacios locales del DA por parte del Estado constituye un reto tanto político como de despliegue de coordinaciones administrativas, principalmente en contextos donde existe un precedente de programas similares que no tuvieron el éxito esperado, pues daña la confianza y limita las expectativas hacia una nueva intervención ${ }^{3}$.

Aguaytía es una de las localidades donde se implementaron con anterioridad programas de DA a través de organismos internacionales y del Gobierno regional. Si bien a nivel nacional Aguaytía —ubicado en el departamento de Ucayali- concentraba el año 2015 solo el 0,5\% del total de hectáreas de coca ${ }^{4}$, desde el año 2013 se viene implementando un programa piloto de DA liderado por el Estado como política a nivel central ${ }^{5}$. Lo que hace a Aguaytía una zona especial es su constitución como punto de encuentro de dos zonas cocaleras (Tocache y el valle del Monzón) ${ }^{6}$, contando con un gran flujo migratorio de

\footnotetext{
3 Esto tanto por parte de los implementadores del proyecto (que no consiguieron alcanzar sus metas de reducción de cultivos de hoja de coca e incorporación sostenida de familias participantes) como de la población participante, que no vio plasmada los beneficios que en principio iban a conseguir.

4 Si bien la extensión de cultivos de hoja de coca ha disminuido en los últimos años debido a la erradicación, también se ha dado por una migración de cocaleros a valles aledańos (especialmente Pichis-Palcazú, en Pasco), con quienes se mantiene un comercio frecuente.

5 Este modelo piloto, tanto en sus aciertos como desaciertos, sirvió como espacio de aprendizaje para intervenciones que realizó el Estado en otros valles (como Monzón, Pichis-Palcazú, entre otros).

6 Estratégicamente podría ser concebido como un punto nodal a partir del cual se cree una "barrera» para el narcotráfico.
} 
estos valles, principalmente de agricultores que se fueron asentando mediante apropiación de tierrras.

El proceso histórico de expansión de cultivos de hoja de coca fue un elemento central que configuró desde el principio redes comunitarias en esta zona, se organizaban para evitar que las tierras ganadas y lo sembrado en ellas sean fiscalizadas por el Estado. Es decir, existió en la zona una organización colectiva histórica en contraposición a la entrada de instituciones del Estado (especialmente aquellas relacionadas a la fiscalización).

Los programas de DA enfrentan un mayor reto en Aguaytía debido a la saturación de programas anteriories que no tuvieron éxito. Las múltiples intervencciones pasadas han generado una predisposición negativa hacia este tipo de programas y limitan las expectativas de la población.

Por todos estos factores, el DA es un tema politizado en la zona; forma parte del discuro vertebral de la Organización de Cocaleros, los principales políticos - ya sean líderes o autoridades locales — han captado aprobación y votos demostrando explícitamente su rechazo o apoyo hacia el programa de DA, e incluso la participación en las radios locales en este tema ha sido notorio. ${ }^{7} \mathrm{Se}$ podría postular que, hasta cierto punto, en la última década las negociaciones en torno a la implementación de programas de DA ha sido uno de los ejes centrales de la política local, y algunos actores más que otros lo supieron capitalizar políticamente.

Por los motivos anteriormente expuestos, Aguaytía se fue convirtiendo en un reto por dos razones: por un lado constituye un espacio simbólico de «contención» frente a la propagación de cultivos hacia los valles colindantes; por otro lado, políticamente el desafío se encuentra en enfrentar y remediar concepciones negativas del DA y del accionar estatal.

\subsection{La capacidad relacional del Estado y el reto de la implementación en el contexto: aspectos teóricos}

La forma en la cual entendemos el Estado puede tener múltiples aristas, siendo una de ellas las capacidades que tiene para conseguir sus objetivos de forma eficiente y eficaz (O’Donnell, 2007; Oszlak, 1999). Sin embargo, las capacidades para ejercer como un actor más - y en algunas ocasiones actuar como árbitro- no son suficientes para entender el accionar del Estado si no se considera su capacidad relacional con la sociedad. Guillermo Alonso identifica

\footnotetext{
7 En esta investigación se entrevistaron a tres locutores radiales que eran reconocidos como formadores dentro de la localidad.
} 
dos dimensiones de las capacidades estatales: las técnico-administrativas y las relaciones (Hevia, 2015). Si bien la capacidad relacional se manifiesta en los distintos poderes del Estado, es especialmente notorio en la implementación directa que tiene el Estado a través de sus instituciones en relación con la sociedad. Así, la capacidad relacional es más explícita en el marco de las políticas públicas, ya que se propone que existe una relación entre el Estado y la sociedad para determinar las políticas públicas y la forma en la cual estas son ejecutadas, en pos de lograr sus objetivos en consonancia con lo que la sociedad percibe como cambio positivo (Berreta et al., 2005). Focalizando la mirada en el carácter relacional de la capacidad estatal situada a través de actores concretos, coincidimos en la definición de capacidad estatal de Hevia, según el cual, esta «implica la habilidad de los actores estatales para articular con los actores sociales las políticas públicas, de modo de poder implementarlas con el apoyo de los sujetos sociales/ciudadanos a quienes incumben» (Hevia 2015, p. 125).

Al estudiar el accionar del Estado en países como el Perú, es importante dar cuenta que no se trata de una entidad homogénea ni unitaria. El Estado funciona mediante $-\mathrm{y}$ a pesar de - intereses fragmentados y contradictorios, enfrentando múltiples resistencias a sus intervenciones que resultan en una dinámica de negociación. Escalante, quien estudia el caso del narcotráfico en México, resume las interacciones entre el Estado - y sus objetivos de obtener control territorial y político- y la sociedad civil de la siguiente manera:

El Estado define el campo político, define las normas para regular el conflicto, pero a la vez participa en ese campo político: el Estado define funciones, atribuciones, límites, pero después tiene que intervenir mediante individuos concretos, investidos como autoridades o funcionarios, en el campo social. En el momento de intervenir, los representantes del Estado están inmersos en un sistema de relaciones sociales que no controlan. Su autoridad, sus atribuciones y recursos, pueden usarse hasta cierto punto, porque con frecuencia tienen que negociarse con actores sociales que controlan recursos de distinto tipo (Escalante, 2007, p. 73).

Se reconoce que, en estos contextos (de narcotráfico), el Estado no es el actor que mantiene el absoluto control territorial, así como tampoco plantea una dirección hegemónica del desarrollo y las actividades que se llevarán a cabo para alcanzarlo. Más bien, el Estado se convierte en un actor más que se manifiesta a través de individuos concretos y contextualizados, los cuales deben encontrar un punto de equilibrio entre los objetivos institucionales, los recursos con los que cuentan (personales e institucionales), las necesidades de la población y sus propios intereses. En el caso mexicano, Hevia encuentra 
que existen tres patrones de relaciones entre el Estado y la sociedad: la relación particularista, las relaciones contenciosas-disruptivas y las relaciones de participación reglamentaria (2015, p. 115).

En tanto que estamos frente a un contexto con múltiples intereses y formas de actuar, lo que prima en este contexto es una negociación entre agencias gubernamentales, exigidas a dar soluciones a la pobreza, y la sociedad que actúa al borde de la legalidad en búsqueda de reinvindicaciones sociales parciales (Jungemann, 2010) ${ }^{8}$. Así, las poblaciones en ámbitos periféricos y marginales tendrían una real agencia en la implementación de políticas públicas, ya que se encuentran en la capacidad de exigir y satisfacer demandas concretas (muchas veces por vías paralegales) para conceder, a su vez, la implementación de políticas a funcionarios públicos y permitir que cumplan con sus metas (Chatterjee, 2007, p. 150). En el caso de Aguaytía, la población se encuentra en una situación de pobreza, sumado a la situación adversa de la erradicación, lo que lleva a exigir acciones y soluciones inmediatas para paliar su situación.

Sin embargo, anterior a la implementación directa de una política pública, es fundamental generar un «ambiente autorizado»; es decir, que las iniciativas puedan ser aceptadas y lideradas localmente para que sean sostenibles (Andrews, Pritchett y Woolcock, 2016). No es posible la implementación sostenible de políticas públicas si no se cuenta de antemano con la legitimidad en terreno.

En este caso, la solución que se presenta a la presencia del narcotráfico y a los efectos de la erradicación son los programas de DA. Sin embargo, así como suele suceder en otras políticas públicas, el modelo de DA deviene de experiencias en otros lugares, tanto dentro como fuera del Perú ${ }^{9}$. En el caso de Aguaytía, el modelo de DA liderado por el Estado se implementó de manera muy similar a los anteriores programas -independiente de las instituciones que la lideraban-, sin sufrir mayores cambios en su diseño e indicadores (a pesar de no mostrar señales concretas de avance).

En el mundo de las políticas públicas, es usual la reproducción continua de «proyectos modelo» que no logran aparentemente solucionar el problema, pero que cumplen con los indicadores de avance. Este fenómeno es descrito por Andrews, Pritchett y Woolcock como el isomorfismo mimético, identificado por DiMaggio, el cual se refiere a «la tendencia de introducir reformas que

\footnotetext{
Chatterjee analiza el caso de los asentamientos humanos en India (ilegalmente constuidos) y la forma en la cual la población busca reivindicar derechos sociales (acceso a la vivienda, luz, agua, entre otros) mediante una relación tensionada con los agentes gubernamentales.

9 El caso paradigmático del desarrollo alternativo en Perú se dio en la región de San Martín, donde el modelo construido de DA tuvo éxito en demostrar el avance de la lucha conra el narcotráfico según los indicadores establecidos, constituyéndose así con el nombre de «Milagro de San Martín».
} 
mejoran el apoyo y la legitimidad de una institución externa, incluso cuando no existen pruebas concretas de mejoramiento» (Andrews, Prichett y Woolcock, 2012, p. 6). Así, de acuerdo a estos autores el «entrampamiento en las capacidades estatales» se origina cuando: a) se reproducen soluciones externas al contexto consideradas como las «mejoras prácticas en las agendas»; b) a través de procesos lineales; c) que informan limitados datos en relación al plan original; d) son implementados de forma vertical, asumiendo que la implementación a nivel local se da de manera literal (Andrews, Pritchett y Woolcock, 2012).

Frente a la rigidez del diseño de una política, los actores locales tienen que articular sus esfuerzos para cumplir con sus objetivos. Así, lo que suele suceder en estos casos es que los frontline workers («burócratas de pie») tienen metas estrictas preestablecidas, pero se enfrentan a un contexto que muchas veces sobrepasa las instrucciones recibidas, razón por la cual deben hacer uso de su autonomía, generando tanto desviaciones positivas como negativas (Andrews, 2013). La implementación del programa de DA en Aguaytía no constituye una excepción, sobre todo por el contexto complejo poserradicación de los cultivos de hoja de coca y la predisposición de la población frente a estos programas (anteriormente señalado).

\subsection{La triangulación metodológica para visualizar las relaciones}

Las etapas que constituyeron esta investigación fueron aquellas que propone Bertaux (1989): exploratoria, explicativa y sintética. En primer lugar, se realizó una etnografía exploratoria en base a los datos estadísticos obtenidos de los informes de monitoreo del cultivo de coca de la UNODC y los informes de seguimiento de avance de los programas presupuestales de desarrollo alternativo integral y sostenible (Pirdais) generados por Devida ${ }^{10}$, donde se identificaron las ideas fuerza que nos permitieron definir el enfoque sociológico. En una segunda aproximación a nuestro caso, los datos que se recogieron en el trabajo de campo mediante entrevistas y observaciones de campo sirvieron para validar los ejes de análisis en torno a las diferencias perceptivas de las raíces causas del problema del narcotráfico y sus posibles soluciones. En tercer lugar, con la información obtenida se realizó un análisis sintético respecto a la información estadística emitida por las instituciones que lideran la lucha contra las drogas y la información respecto a las dinámicas generadas directamente en el campo.

Para fines de una mayor rigurosidad, seguimos el método de triangulación metodológica (Denzin, 1990); se realizaron entrevistas estructuradas, entrevistas semiestructuradas y observaciones participantes y no participantes para

10 Ambos tipos de informes están indicados en la bibliografía. 
poder contrastar las fuentes y los ejes de análisis. Para identificar el número de entrevistas que debíamos realizar, fuimos encontrando el "punto de saturación", referente a constatar con diferentes informantes las mismas unidades de análisis, hasta que ciertas nociones se fueron repitiendo (de esta manera sabemos que se trata de un fenómeno colectivo y, por lo tanto, generalizable).

En la implementación de programas de DA intervienen una multiplicidad de actores que incluyen al Estado como ente normativo y actor intervencionista, las familias de agricultores como la población objetivo, los distintos niveles de gobierno, las autoridades comunales, y la cooperación internacional. Por esta razón, se entrevistaron a los siguientes tipos de actores:

- Personal de Devida: principalmente los «socializadores» (encargados de presentar el programa de DA en las poblaciones) y técnicos agrónomos (quienes acompañan en el cambio de cultivo)

- Líderes de la Organización de cocaleros ${ }^{11}$

- Líderes de opinión local

- Agricultores, tanto participantes como no del programa de Desarrollo Alternativo

El proceso de investigación en campo se realizó entre los años 2012 y 2014 en Huipoca-Aguaytía. Si bien se visitó casi la totalidad de los caseríos que conforman el Centro Poblado Huipoca, las entrevistas se realizaron principalmente en los caseríos de Shanantía y Nuevo Progreso, ya que eran localidades reconocidas por su resistencia a los programas de DA y se tuvo la oportunidad de acompañar en las negociaciones.

Para realizar el trabajo de campo se generó un protocolo de consentimiento informado respecto a la investigación. Los participantes (entrevistados) fueron informados respecto a los fines de la investigación y se les entregó el correo de la investigadora para contactarla ante cualquier duda. Para mantener la confidencialidad e integridad de los participantes de esta investigación, se presentarán de forma anónima.

\section{LA IMPLEMENTACIÓN DEL PROGRAMA DEL DA: DIVERGENCIAS LOCALES Y AJUSTES NECESARIOS}

Podemos identificar dos etapas de acercamiento y relacionamiento entre la población local y los actores encargados de implementar el DA. En estas dos

11 El objetivo de estas entrevistas era analizar cuál era la visión que tienen en torno al programa de Desarrollo Alternativo, para evaluar el grado de influencia que tienen en la respuesta de los agricultores. 
etapas las interacciones no son meramente expositivas, sino que se constituyen como campos de negociación de acuerdos, de división de responsabilidades e inclusive de construcción de significados en torno a lo que se espera del desarrollo que promete el DA.

En la primera etapa se realiza una presentación personal del socializador en su rol dentro del programa de DA y las características de este último. Por su naturaleza, se trata de un primer encuentro para generar confianza a través de la contención de posibles quejas y las respuestas tranquilizadoras frente a ellas, reconociendo errores anteriores y explicitando el compromiso de no repetirlos en una segunda ocasión. Estos primeros acercamientos se realizan tanto de manera individual (visitando a cada potencial familia participante) como de manera grupal (en asambleas comunales). Sin embargo, la práctica más común es reunirse con cada posible participante de manera individual, de manera tal que no estén presentes posibles actores que puedan generar un discurso líder opositor frente a los programas de DA (porque estos no serían beneficiosos para la población).

¿Por qué el Estado necesita generar una fragmentación en los procesos de negociación e intervención comunitaria? Con antelación a la entrada del Programa de Desarrollo Alternativo de Devida, las comunidades formaban un bloque de opinión y de oposición relativamente homogéneo, siendo uno de los puntos de unión evitar la entrada del Estado. La oposición frente al Estado se basa en la percepción de que no es un ente que genera bienestar y desarrollo (a través de servicios sociales), sino que se manifiesta a través de políticas restrictivas y punitivas (como la erradicación). Según un agricultor de la zona:

El tema del PDA... para mi concepto se ha caracterizado siempre por ser una de las ONGs más mentirosas que pudo haber existido en Aguaytía. Eeh, porque lo que ellos buscaban era desestabilizar a un caserío porque era la única forma en que podían ingresar (Agricultor 2, Caserío Nuevo Progreso).

Más allá de la conceptualización del DA -y las confusiones respecto a la naturaleza de la organización que la implementa-, este testimonio es elocuente en mostrar que la entrada se realiza a través de la ruptura de la posible respuesta en bloque. Actuando bajo la prenoción de que no existe capital social «lícito» ${ }^{12}$, sino que este se constituye a partir del narcotráfico - y, por tanto, en una relación negativa con el Estado_-, la estrategia que utilizan los socializadores

12 Pareciera reconocerse en la ENLCD que el capital social solamente puede ser entendido como tal cuando se genera en contextos lícitos y relación armoniosa con el Estado, y no tanto como un campo de relaciones sociales (independientemente que surja en un contexto de economía ilícita). 
es convencer a cada agricultor de forma individual, sin considerar que la participación de algunos agricultores y la exclusión de otros puede generar conflictos en la comunidad. Esta estrategia se encuentra legalmente justificada ${ }^{13}$ y concuerda con lo identificado por Chatterjee (2007) con respecto a la relación Estado-sociedad en países postcoloniales; se identifica al ciudadano bajo una mirada individualista y universal, sin considerar que hay formas de organización preexistentes a esta concepción del ciudadano universal del Estado moderno.

De esta manera, la estrategia fragmentadora de Devida no es gratuita; al convencer en forma individual la participación de los agricultores, lo que se genera es una profundización de la individualización de relacionamiento con instituciones externas a la comunidad y, por lo tanto, anula la posibilidad de que la oposición a los programas de DA se consolide en un bloque a nivel de comunidad.

La mayoría de los agricultores entrevistados aseguraban que, con la entrada de Devida, la comunidad se había vuelto más desunida y disgregada.

Y por eso es que yo estoy ahorita participando en este proyecto, porque yo no puedo ser parte de lo que he criticado más antes como dirigente y ahora me contradiga en estar trabajando. Como muchos me están cuestionando que yo soy traidor, pero digo "¿qué cosa he traicionado?» (Agricultor 4, Caserío Shanantia).

La etapa culminante de las fases de negociación es la «Firma de Acta de Entendimiento», que sería la segunda etapa del proceso de negociación. El acta significa el compromiso del Estado en el desarrollo productivo dentro de las comunidades, con el compromiso de la población de no extender sus cultivos de coca. La responsabilidad de responder frente al Estado de parte de la comunidad viene dada por alguna autoridad comunal, y en cada comunidad están presentes, mínimo, dos figuras de autoridad: el agente municipal (como representante del municipio) y el teniente gobernador (representante del gobierno).

Para que pueda firmarse el Acta de Entendimiento se requiere que una de las autoridades comunales esté de acuerdo con el compromiso con Devida. Si bien lo ideal es que ambas figuras concuerden con la entrada de Devida, si una de ellas está en desacuerdo, se trabaja indistintamente con la autorización de la otra. Esto deriva que, a través del Estado, las autoridades comunales pierdan legitimidad y se produzca un resquebrajamiento en la división de poderes y responsabilidades al interior de las comunidades. Esta estrategia se vuelve

13 Mencionamos que está legalmente justificada en tanto que no es un actividad prohibida en los lineamientos del DA. 
eficiente debido a la consonancia que genera en los intereses particulares de los actores locales (especialmente aquellos que tienen una posición de poder en las comunidades) ${ }^{14}$.

Así, se capitaliza la relación con el Estado (institución considerada inicialmente como «externa») como forma de ganar legitimidad dentro del caserío, pero con el compromiso tangible de parte del Estado que los beneficios que se obtendrán —en torno al acceso a servicios, posible reconocimiento del Estado como asociaciones modelo, entre otras - serán reales. Esto, debido a que, si bien la autoridad que confirma su predisposición de trabajar con Devida lo hace en tanto representante legal de la misma, también generalmente resuena con sus propios intereses de incrementar su capital político mediante la exigencia de Devida de cumplir con sus compromisos.

En síntesis, como estrategia para hacer frente a la respuesta confrontacional basada en una percepción negativa respecto al Estado, la forma que este tiene de intervenir en una comunidad es intentando resquebrajar las redes de apoyo que se han constituido a partir del narcotráfico ${ }^{15}$ a través de la participación individualizada y dividiendo la legitimidad de las autoridades comunales. Es interesante que, finalmente, la relación que busca tener el Estado con los agricultores no sea a través del fortalecimiento de organizaciones de base, lo cual repercute posteriormente en actividades que buscan fomentar la cohesión en el programa de DA, tales como la constitución de juntas vecinales, asociaciones productivas, entre otras.

\subsection{El desplazamiento de responsabilidades de efectos adversos como estrategia de flexibilización y la flexibilización en torno a la siembra de cultivos de coca}

Para lograr una cercanía con los diversos actores que participarán en el programa de DA, los socializadores y otros agentes gubernamentales deben generar diversas estrategias que les permitan su entrada y mantenimiento en las comunidades. La individuación de las relaciones entre las personas de la comunidad y el Estado es una, pero que se ve complementada con dos estrategias claves: la disociación de otras instituciones ligadas a la lucha contra el narcotráfico

\footnotetext{
14 Chatterjje realiza un análisis de demandas sociales en barrios, demostrando que en la interacción y negociación de demandas, los líderes de la sociedad política también tienen intereses particulares para aumentar su grado de influencia.

15 Como hemos mencionado anteriormente, debido principalmente a que las comunidades se constituyeron en esta localidad mediante la migración de agricultores — principalmente de hoja de coca— de valles aledańos donde la práctica del cultivo era regular y más antigua.
} 
(como el Corah del Ministerio del Interior) y la flexibilización de la normativa en la siembra de la hoja de coca. Estas dos líneas estratégicas toman mayor relevancia si uno contempla que se producen mediante la capitalización de los intereses particulares de cada actor, los cuales, a su vez, logran el objetivo final que es generar estabilidad en la intervención.

Una de las principales acusaciones que tienen los pobladores hacia los trabajadores de Devida es que el Estado no brinda el apoyo necesario y solamente interviene a través de acciones de interdicción (erradicación de cultivos de hoja de coca). En la medida en que Devida postula presentarse como un organismo que dota de oportunidades y evita enmarcar sus acciones en posturas radicales, utiliza la estrategia de desplazar esa responsabilidad al Ministerio del Interior (a pesar de ser ambos representantes del Estado), para así ocultar el rechazo hacia el cultivo de coca y permitir un acercamiento de los miembros a la población. Esta estrategia de posicionamiento de Devida ante la población local es funcional en tanto les ayuda a presentarse como un actor más benigno para la población local, evitando el estigma que podría traer una asociación con la acción de erradicación. Esto guarda relación con lo mencionado por Andrews, Pritchett y Woolcock (2016), quienes señalan que para que una intervención sea sostenible y se transforme en una noria de cambios, es central generar un «ambiente autorizador». En este ambiente autorizador generalmente están presentes diversos actores en diferentes niveles (de la misma u otra organización) que ejercen controles distintos sobre los mismos procesos.

Es curioso que las divergencias respecto a la toma de responsabilidades dentro del Estado en torno a la lucha contra el narcotráfico se canalicen como recurso para lograr un distanciamiento respecto a acciones que generan rechazo a la población. De esta manera, se logra producir un acercamiento entre los agricultores y las instituciones asociadas a desarrollo. Esto lo podemos ver claramente en los testimonios de dos socializadores:

Pero ahora yo les digo: hermanitos, yo no necesito condicionar porque el Estado sí o sí va a venir a erradicar ${ }^{16}$, entonces yo me doy la libertad ya de decirles así: mi problema es simplemente traer desarrollo, empezando por el cacao, mańana te puedo traer otro producto, pasado te traigo otro, pasado voy a gestionar contigo, vamos a traer a tal persona, y es verdad (Socializador 3).

16 La cursiva es nuestra. 
Yo creo que el gobierno que hace la erradicación por medio del Ministerio del Interior, es una cosa de gobierno de Estado, y nosotros ya vamos como quién dice no tenemos nada que ver en el asunto, ${ }^{17}$ una cosa así ¿no? (Socializador 5).

Una razón importante por la cual se genera un distanciamiento con otras instituciones vinculadas con la lucha contra las drogas tiene que ver con la estabilidad y legitimidad de la intervención. Las políticas de interdicción son programadas desde el Gobierno central y se realizan mediante grupos especializados $^{18}$ a través de operativos de manera puntual. De esta manera, los responsables de llevar a cabo estos operativos de erradicación no tienen la necesidad de quedarse en las localidades y generar un vínculo con la población. Contrariamente a esta lógica, el DA contempla años de desarrollo, lo que deviene en que las únicas vías para tranzar con las comunidades y generar gobernabilidad sean las interacciones entre los agentes gubernamentales del DA y los agricultores.

La acción de trasladar responsabilidades a otras instituciones del Estado es también funcional y congruente con el delineamiento de metas administrativas divididas entre las instituciones que coordina Devida; mientras que el Ministerio del Interior tiene metas de erradicación de cultivos de hoja de coca, Devida tiene como meta la incorporación de familias ex cocaleras al DA.

La escisión de responsabilidades y distintas percepciones de lo que debe hacerse respecto a la lucha contra las drogas no solamente se presenta a nivel central entre las diferentes instituciones estatales y no gubernamentales, sino también a nivel de los diferentes niveles de gobierno. A nivel de Gobierno local la voluntad política de implementar políticas de DA encuentra también disonancias, debido especialmente a experiencias anteriores que, a los ojos de la población local, no tuvieron éxito por un incorrecto diagnóstico. De esta manera, la visión hegemónica de lo que debe realizarse en términos de lucha contra las drogas encuentra resistencia no solo a nivel de comunidades, sino también a nivel de autoridades regionales. En este aspecto, lo que prima es una característica similar a la identificada por Hevia en México, en la relación denominada como "particularista», según la cual «los gobernantes pueden capitalizar políticamente las decisiones y el presupuesto que manejan, permite utilizar la gestión como instrumento de castigo/premio, genera lealtades y promueve la densidad de relaciones de reciprocidad para con los beneficiarios» (Hevia, 2015, p. 119-120). Así, lo que se genera es una relación de negociación

\footnotetext{
17 El subrayado es nuestro.

18 Como el Corah (Cuerpo Operativo Alto Huallaga), una división del Ministerio del Interior encargada de los operativos de erradicación de la hoja de coca.
} 
(legítima) entre los alcances en decisiones que puede tener la Municipalidad distrital y el Gobierno central, pero donde el Gobierno central maneja mayor campo de decisión, al poder realizar excepciones cuando lo estime conveniente (Crozier, 1969). En este caso, lo que se genera es un espacio de diálogo entre los distintos niveles de autoridades para determinar (formal e informalmente) los términos de la intervención, especialmente en lo que respecta a las plantaciones de cultivos coca.

Sin embargo, ¿̇en torno a qué se realizan las negociaciones en el marco de implementación del DA? En el caso peruano, los programas de DA se implementan después de episodios de erradicación forzosa de los cultivos de hoja de coca (a pesar de las numerosas críticas que tiene esta secuencia) ${ }^{19}$. El único valle en el cual se viene implementando una secuencia distinta a partir de 2015 es el valle de los ríos Apurímac, Ene y Mantaro (donde se concentran la mayor cantidad de cultivos de hoja de coca).

A pesar de todas las previsiones, la erradicación representa una decisión inalterable que, inevitablemente, perjudica a los agricultores puesto que se quedan sin sus sustentos básicos. Por esta razón, es muy importante considerar como un elemento central la precondicionalidad de erradicación de cultivos de coca para ser participante del programa de DA, ya que de parte de los agricultores la amenaza de la erradicación implica una latente frustración, mientras que por parte de los trabajadores del Estado el escenario de post erradicación les deposita la responsabilidad de ofrecer una alternativa adecuada.

Según la normativa institucional, la postura de los trabajadores de Devida debería ser la de un rechazo contundente a la siembra de hoja de coca. Sin embargo, a pesar que el Estado condiciona la participación de los agricultores en el DA con la exigencia del abandono de los cultivos de coca, lo que ocurre generalmente es que los agricultores mantienen ambos cultivos, traslapando diferentes lógicas de producción agrícola. Esto ocurre bajo la mirada permisiva del personal de campo de Devida. ¿Por qué los trabajadores de Devida incurren en estas prácticas infractoras? A pesar de que va contra los lineamientos básicos de la institución, este tipo de acciones transgresoras se convierten en funcionales.

Como una forma de enfrentar la toma de decisión unilateral de erradicación de los cultivos de hoja de coca, los socializadores reconocen en su accionar cotidiano que esta situación es contraproducente para incentivar el desarrollo local y forjar confianza con instituciones del Estado. Asimismo, aceptan que el mantenimiento del cultivo de coca a la par de los cultivos lícitos evita el colapso

19 Andersson (2006), Chouvy (2012) y Schultze-Kraft y Befani (2014). 
de la economía familiar que conlleva la erradicación total, y permite una simbiosis económica entre lo lícito e ilícito, hasta que los nuevos cultivos lleguen a la etapa productiva. Los cultivos alternativos tardan aproximadamente de dos a tres años en empezar a brindar sus frutos y ello es sabido por todos los técnicos de campo, razón por la cual permiten que los agricultores subvencionen el crecimiento del cultivo alternativo con las ganancias obtenidas a partir de la cosecha de la hoja de coca. Como menciona un agricultor ${ }^{20}$ :

[...] y por otro lado está diciendo: «siembra tu coca, siembra tu coca», incentivaban a sembrar. Porque ellos mismos sabían que si la gente no tenía coca, el proyecto se iba al fracaso (Agricultor 2, Caserío Shanantía).

Este mantenimiento es también posible debido a que la larga intervención del Estado a través del Ministerio del Interior ha dejado como aprendizaje en las comunidades el conocimiento del funcionamiento de este; la mayoría de los agricultores perciben las temporadas en las cuales se darán los procesos de erradicación, así como también las localizaciones más frecuentes.

En línea con el punto anterior, otra de las adaptaciones que generan los agricultores frente a la erradicación es la combinación, en las parcelas, de cultivos lícitos intercalados con cultivos de coca (como una forma de camuflaje), de manera tal que puedan pasar desapercibidos en el monitoreo que realiza Devida. Si bien esta estrategia realizada por los agricultores socava la política de erradicación, en realidad se encuentra articulada con la flexibilización de la prohibición de la coca que, en la práctica, realizan los socializadores. Además, una consecuencia no deseada — pero de naturaleza positiva — es que el traslape de cultivos lícitos e ilícitos termina, a fin de cuentas, instaurando en los agricultores una dependencia del cultivo alternativo y una habituación a su sembrado.

La crítica implícita realizada por los trabajadores estatales y socializadores respecto al proceso de erradicación y tiempo de espera en ver los resultados concretos del DA, radica no solamente en la problemática del tiempo agrícolamente improductivo, sino también en el riesgo que genera el monocultivo que vino fomentando el DA.

No puede tener solamente un cultivo, tiene que tener de todo el agricultor. Ese el riesgo de los agricultores, ese el riesgo de los cafetaleros, cuando los cafetaleros baja el precio, están desesperados. Si ellos tuvieran otra alternativa frutal para por lo menos afrontar la situación difícil de ese año, de esa campaña, entonces son cosas que hay que ver, ellos dependen de la economía de campo y hay que buscar eso; diversificar porque tienen varias alternativas (Socializador 4).

20 El comentario de este agricultor se pudo constatar con las acciones registradas en la etnografía y con material fotográfico, ya que no se permitió tener una grabación dentro de estas negociaciones. 
Fotografía 1. Platanales del programa de DA intercalados con cocales

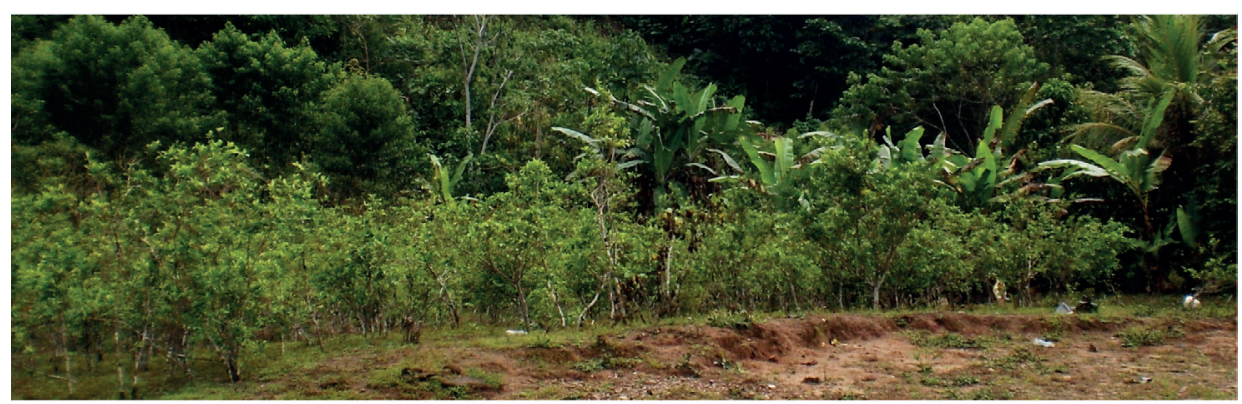

Fuente: propia. Cinturón Centro Poblado Huipoca.

En este sentido, la aceptación de la flexibilización de los cultivos de hoja de coca también está relacionada con el reconocimiento del potencial peligro de la siembra de un solo cultivo (ya sea con respecto a la hoja de coca o con respecto a los cultivos de cacao y café $)^{21}$.

Por esta razón, postulamos que esta acción frente a otras instituciones que trabajan en la lucha contra el narcotráfico (pero en otras líneas) genera estabilidad de la intervención. Es decir, en la práctica, los trabajadores de Devida permiten un proceso de erradicación paulatino o escalonado que contradice la directiva oficial, puesto que su trabajo se sustenta en un compromiso por un proyecto de más largo aliento, que solamente es posible en la medida en que generen una relación de confianza con la población local y construyan un ambiente autorizador legítimo respecto a los avances comprometidos del DA.

Por último, es esencial entender la estabilidad de la intervención no solo desde la perspectiva de la institución estatal, sino también a través de los ojos de los que lo ejecutan: los socializadores. Estos también presentan intereses particulares de mantener la intervención, ya que los indicadores de su avance están determinados a priori y a un mediano/largo plazo.

Por esta razón, no debemos de perder de vista que los agentes gubernamentales también tienen intereses particulares, especialmente porque su trabajo se rige sobre la base de metas establecidas en una lógica de presupuesto por resultados. Si la forma en que se mide el desempeño individual de los socializadores es a través de la cantidad de familias que se inscriben y mantienen en los programas de desarrollo alternativo y las hectáreas de coca que son reemplazadas

\footnotetext{
21 El Estado peruano aprendió de estas experiencias y las nuevas intervenciones que se realizan en el valle del Monzón a partir de 2013 contemplaron biohuertos para el aprovisionamiento temporal.
} 
por cultivos alternativos, tiene sentido pensar que se necesita flexibilizar para llegar a esos acuerdos.

Un agente encargado de negociar con las comunidades, perteneciente a la cooperación internacional, reflexiona respecto a las exigencias de las metas institucionales de los agentes gubernamentales (Devida) y comenta:

El hecho de que tengas metas físicas te obliga a hacer, cumplir las metas, más están preocupados en cumplir las metas del proyecto, que las soluciones del agricultor, las metas del productor, las expectativas del agricultor (Socializador, cooperación internacional).

Evidentemente, no forma parte de la política formal utilizar mecanismos de negociación, pero estos se vuelven necesarios y funcionales debido a los intereses particulares con que juegan los actores estatales, que corresponden a su vez a un planteamiento inadecuado de medir su desempeño y el avance del programa de DA.

\section{Conclusiones}

El debate sobre las capacidades estatales ha sido amplio, especialmente en torno al alcance funcional y territorial del Estado. En el análisis de las políticas públicas es esencial estudiar la capacidad relacional que tiene el Estado para generar proyectos que resuenen en necesidades de la sociedad, permitiendo una relación fluida y que las intervenciones concretas gocen de legitimidad.

Esta no es una tarea fácil si consideramos que el Estado y la sociedad no son entes homogéneos ni unitarios, sino que representan intereses fragmentados tanto dentro de una misma organización —en distintos niveles de jerarquía- como también entre distintas organizaciones que, por voluntad o por imposición, se encuentran dentro de un mismo proceso de implementación. Esto ocurre, por ejemplo, en la zona de Aguaytía, donde existen organizaciones sociales de base productivas, principalmente defensoras de la coca, que por su naturaleza se encuentran inherentemente vinculadas al proceso de implementación de DA. Asimismo, las autoridades locales que deben entablar un punto de equilibrio entre las exigencias de la población local y los lineamientos a nivel central se ven retadas en asumir una posición y liderazgo que sea percibido como positivo por la población. Así, construir puentes de negociación en intervenciones locales, que se ajusten a demandas concretas, se vuelve una actividad fundamental que orienta a construir ambientes autorizados legítimos.

Un desafío esencial de la implementación en Aguaytía nace en la constitución de las comunidades en la zona, que son principalmente migrantes cocaleros 
de otros valles, asentados generalmente de manera ilegal (mediante la apropiación de tierras). Esto determina una resistencia general a relacionarse con el Estado, debido a que ven a este como un agente controlador y punitivo (como se expresa en las acciones concretas de erradicación de hoja de coca o la negativa de otorgar créditos debido a la falta de título de tenencia de tierra), antes que como un articulador de plataforma de servicios.

Las concepciones que tienen los pobladores respecto al accionar del Estado constituyen un repertorio conocido por parte de los agentes gubernamentales - generalmente debido a que tienen experiencias previas en proyectos iguales o similares_, el cual movilizan para poder compatibilizar con la población local, construyendo acuerdos y discursos que no necesariamente representan los ideales de la institución, más si ayudan a su fin superior. Así, el grado de autonomía que tienen los agentes gubernamentales que tratan directamente con los agricultores les abre una puerta para negociar de acuerdo a necesidades prioritarias de la población local. Cabe señalar que, si bien existen organizaciones de productores, de cocaleros y partidos políticos locales, las negociaciones no suelen tener un carácter ideológico, sino más bien se plantean alternativas fuera de la normativa, que buscan darle legitimidad y sostenibilidad al programa.

Ahora, ¿por qué resultan tan necesarias las negociaciones y adaptaciones al contexto local? En la investigación encontramos que esto sucede por dos razones principales: por un lado, por una naturaleza del diseño de política y de seguimiento con alto grado de rigidez (que impide ser modificado en la consulta con la población intervenida), y, por otro lado, porque los actores que están involucrados en el proceso (ya sean gubernamentales como de la sociedad política) tienen intereses particulares y situados, que pueden agilizar o entorpecer el proceso.

Con relación al diseño de la política, lo que se sugiere es que se reproducen ideas en torno a lo que genera el narcotráfico, extrapolando casos exitosos, pero generalmente en forma y no en contenido. La pregunta que nos surge en este caso sería: ¿por qué la forma de entender el narcotráfico se mantiene en la forma en que lo hace? Existen diversas teorías que pueden explicar el mantenimiento de la estrategia como se está llevando a cabo, pero nos inclinamos por apostar que se daría básicamente por una lógica de isomorfismo mimético. Asimismo, ocurren todos los elementos que identifican Andrews, Prichett y Woolcock (2012) como el «entrampamiento en las capacidades estatales» cuando: a) se reproducen soluciones externas al contexto consideradas como las «mejoras prácticas en las agendas»; b) a través de procesos lineales; c) que informan limitados datos en relación al plan original; d) son implementados 
de forma vertical, asumiendo que la implementación a nivel local se da de manera literal. Efectivamente, en el caso del programa de DA en Aguaytía se reprodujeron mejores prácticas (como el ejemplo del Milagro de San Martín) a través de un proceso lineal (ausente de una retroalimentación activa e iterativa de parte de los actores locales), informando limitados datos en relación al plan original (de propulsar desarrollo como una medida preventiva al narcotráfico) e implementando de forma vertical (asumiendo que en la práctica se realizaría de forma literal pero, según lo investigado, no es lo que finalmente ocurría).

Esto lleva a que formalmente se cumplan con los indicadores, pero en base a actuaciones irregulares de los agentes gubernamentales. Lo anterior confluye con el hecho de que la organización de la gestión de la política pública en el Estado peruano se da actualmente a través de una lógica de presupuesto por resultados (PpR). El PpR se refiere al financiamiento de las actividades dependiente de un planeamiento estratégico de metas financieras correlacionadas con metas de actividades (de acuerdo al cumplimiento de las últimas se predispone el presupuesto). Esta mirada se basa en el nuevo institucionalismo, donde se postulaba que la forma de fortalecer al Estado era mediante un control más robusto de los procedimientos y control presupuestario. Sin embargo, cabría preguntarnos si en estas circunstancias no sería más adecuado y eficiente un enfoque en torno a la nueva gestión pública (NPM por sus siglas en inglés para New Public Management). En esta perspectiva, se plantea el Estado como un gestor, un actor líder, que debe proporcionar los incentivos adecuados para que sean los propios actores quienes generen soluciones a largo plazo en torno a su propio beneficio. ¿Cuál sería la diferencia entre este enfoque y el enfoque tradicional? Según Arellano, que:

Al estar el Estado compuesto por actores interesados, la eficiencia solo podrá garantizarse si se generan reglas del juego que liberen la acción de los actores sociales en todo su potencial y permitan controlar a los actores políticos y gubernamentales para dirigir sus esfuerzos hacia los resultados y no hacia su propia agenda política (2007, p. 98).

En este sentido, los instrumentos que se construyen para evaluar los resultados de las políticas públicas no devienen de un acto puramente técnico sino también político, en tanto la consecución de objetivos tiene que ser considerada necesaria y lógica por los actores involucrados.

Las negociaciones generadas en esta zona se dan a partir de la individualización de la respuesta en las comunidades. La división de poderes en las comunidades y la creación de distintos intereses hacen que el tejido comunitario originado en base al narcotráfico se fragmente y permita una entrada soslayada y lenta de la presencia del Estado. Asimismo, otra estrategia central 
en la implementación del programa de DA es el desplazamiento de responsabilidades hacia otras instituciones del Estado. Ambas estrategias (de individuación de la respuesta y el desplazamiento de responsabilidades) generan lo que mencionan Andrews, Pritchett y Woolcock (2016) como un «ambiente autorizador»; quiere decir que la institución genera una relación directa con los diversos actores, con el fin de que la intervención sea sostenida y goce de legitimidad.

En este punto sería necesario seguir cuestionándonos hasta qué punto es beneficioso para el Estado buscar asentarse en estas zonas a través de un incremento del capital político en un segmento pequeño, en desmedro del capital social que ya se ha conformado. Como se ha mencionado, si bien el capital social se conformó a partir del narcotráfico, no por ello termina siendo menos legítimo. Además, es sobre la base de esta organización sobre la cual debería actuarse, incentivando la consecución de los objetivos institucionales como un beneficio más, antes que como una precondición restrictiva.

No debe perderse de vista que la implementación de proyectos se da en campos llenos de significados, con actores particulares y con diferenciales de poder, donde el Estado es uno más de los actores que, además, se sitúa a través de actores particulares. De esta manera, lo primordial es la interacción cara a cara —más allá de los lineamientos y restricciones institucionales—, espacio en el cual finalmente se puede negociar y equilibrar intereses y expectativas. Empero, la tarea pendiente se centra en poder plasmar estas estrategias colaterales «hacia arriba»; es decir, recoger la experiencia de los stakeholders para redefinir la enunciación de la problemática del narcotráfico y del DA, teniendo como consecuencia una flexibilización en la ENLCD con indicadores y objetivos más realistas. Una alternativa plausible sería también generar la implementación de los programas a través de la metodología de intervención y evaluación denominado Problem Driven Iterative Adaption (Adaptación Iterativa enfocado a problemas, desarrollado por el Centro para el Desarrollo Intenacional, Universidad de Harvard), el cual plantea una lógica de determinar cuáles son los principales problemas con los actores principales, y los recursos necesarios para resolverlos mediante una intervención que goce de legitimidad y aceptación. 


\section{REFERENCIAS}

Alonso, G. (2007). Capacidades estatales, instituciones y politica social. Buenos Aires: Prometeo.

Andersson, C. et al. (2006). Impact Assesment of Crop Erradication in Afghanistan and Lessons Learned from Latin America and South East Asia. Contributions to the Kabul International Winter Symposium on Drug Policy. Londres: MF Publishing Ltd.

Andrews, M. (2013). Do international organizations really shape government solutions in developing countries. Boston: Center for International Development at Harvard University.

Andrews, M., Pritchett, L. y Woolcock, M. (2012). Looking like a state: Techniques of Persistent Failure in State Capability for Implementation. Boston: Center for International Development at Harvard University.

Andrews, M., Pritchett L. y Woolcock, M. (2016). Managing your authorizing Environment in a PDIA Process. Documento de trabajo. Boston: Center for International Development at Harrvard University.

Arellano, D. (2007). Una cuestión de confianza. Retos e instrumentos para una reforma del Estado en América Latina. Nueva Sociedad, (210), 95-109. Recuperado de: https:/www.insumisos. com/lecturasinsumisas/Reforma\%20del\%20Estado\%20en\%20America\%20Latina.pdf

Berreta, N. et al. (2005). Transformación estatal y gobernabilidad en el contexto de la globalización: el caso de Uruguay. Capacidaddes estatales aplicadas a la generación de valor agregado en las políticas públicas en Uruguay. Montevideo: Centro de Investigaciones Económicas (CINVE).

Bertraux, D. (1989). Los relatos de la vida en el análisis social. Rev. Historia de antropología y Fuentes Orales. Historia y Fuente Oral, 1(1), 87-96.

Chartterjee, P. (2007). La nación en tiempo heterogéneo y otros estudios subalternos. Lima: EIP, CLACSCO, SEPHIS.

Chouvy, P.A. (2012). A tipology of the unintended consequences of Drug Crop Erradication. Journal Of Drug Issues, 43(2), 216-230. https://doi.org/10.1177/0022042612467989

Crozier, M. (1969). El fenómeno burocrático: ensayo sobre las tendencias burocráticas de los sistemas de organización modernos y sus relaciones con el sistema social y cultural (Vol. 2). Buenos Aires: Amorrortu.

Denzin, N. (1990). Triangulation. En J. Keeves, Educational Research, Methodology and Measurement. An International Handbook. Oxford: Pergamon Press.

Dimaggio, P. y W. Powell. (1983). The iron cage revisited: institutional isomorphism and collective rationality in organizational fields. American Sociological Review, 48(2), 147-160.

Drogas, C. N. (2012). Estrategia Nacional de Lucha Contra las Drogas 2012-2016. Lima: Devida.

Escalante, F. (2007). Baile de máscaras: conjeturas sobre el Estado en América Latina. Nueva Sociedad, (210), 64-77.

Evans, P. (1996). El Estado como problema y como solución. Desarrollo Económico, 36(40).

Hevia, F. (junio de 2015). Construcción de capacidades estatales y patrones de relación Gobiernociudadanos en México: un análisis del nivel subnacional. Revista del CLAD Reforma y Democracia, (62), 107-134.

Hevia, F. y Vera I. (2010). La perspectiva de interfaz aplicada a las relaciones sociedad civil-Estado en México. En A. Olivera (ed.), La democratización frustrada: limitaciones institucionales y colonización politica de las instituciones garantes de derechos y de participación ciudadana en México. Ciudad de México: Centro de Investigaciones y Estudios Superiores en Antropología Social: Universidad Veracruzana.

Jungemann, B. (septiembre-diciembre de 2010). Reseña a "La nación en tiempo heterogéneo y otros estudios subalternos" de Partha Chatterjee. Cuadernos del CENDES, 27(75), 155-159. 
O’Donnell, G. (2007). Hacia un Estado de y para la democracia. En R. Mariani (coord.), Democracia/Estadolciudadanía: hacia un Estado de y para la demoracia en América Latina (pp. 25-64). Lima: PNUD.

Oszlak, O. (1999). De menor a mejor: el desafío de la segunda reforma del Estado. Nueva Sociedad, (160), 81-100.

Oszlak, O. y Orellana, E. (2001). El análisis institucional: aplicación de la metodolog\{ia SADCI. Buenos Aires: Mimeo.

Repetto, F. (2003). Capacidad estatal: requisito necesario para una mejor política social en América Latina. VIII Congreso Internacional del CLAD sobre la Reforma del Estado y la Administración Pública. Panamá.

Schultze-Kraft, M. y Befani B. (2014). Getting high on impact: the challenge of evaluating drug policy. Policy Brief 3. Global Drug Policy Observatory.

Stockli, G. (2015). Estrategias de negociación entre el Estado peruano y los cocaleros organizados: el caso del Aguaytía, Ucayali. VII Congreso Latinoamericano de Ciencia Política. Lima: PUCP..

\section{Fuentes estadísticas}

Devida-Comisión Nacional para el Desarrollo y Vida Sin Drogas (2014). Encuesta de Impacto Desarrollo Alternativo. Lima: Devida.

Devida (2013). Encuesta de Impacto Desarrollo Alternativo. Lima: Devida.

Devida (2012). Encuesta de Impacto Desarrollo Alternativo. Lima: Devida.

UNODC-Oficina de las Naciones Unidas contra la Droga y el Delito (2016). Perú. Monitoreo de Cultivos de Coca 2015. Lima: UNODC

UNODC (2015). Informe Mundial sobre las Drogas 2014.

UNODC (2014). Perú. Monitoreo de Cultivos de Coca 2013. Lima: UNODC. 